\title{
Adaptive quasi-dynamic state estimation for MV and LV grids
}

\author{
Natallia Makarava* (D), Guosong Lin and Sascha Eichstädt
}

\begin{abstract}
State estimation in middle- (MV) and low-voltage (LV) electrical grids poses a number of challenges for the estimation method employed. A significant difference to high-voltage grids is the lack of measurements as the instrumentation with measurement equipment in MV and LV grids is very sparse due to economical reasons. Typically, pseudo-measurements are used as a replacement for actual measurements to this end. A recently proposed disturbance observer based on the extended Kalman filter uses a simplified dynamic model for the errors in the pseudo-measurements of bus power. The aim is then to estimate the errors in the pseudo-measurements and thereby improving the overall estimation result. Despite initial promising results of this so-called nodal load observer (NLO), the main disadvantage of this method is the need for a suitable dynamic model for the error of the pseudo-measurements. Therefore, we here propose a versatile dynamic model for the disturbance observer based on autoregressive processes (AR). We consider a recently proposed online learning algorithm for the prediction of the AR model parameters together with the extended Kalman filter disturbance observer. We demonstrate that this approach results in an efficient method for the dynamic state estimation for MV and LV grids than the original NLO method.
\end{abstract}

Keywords: Kalman filter, Dynamic state estimation, Nodal load observer, AR processes

\section{Introduction}

Static state estimation is a standard procedure in power network analysis to obtain information at all system buses at a given time point [1]. That is, a single set of measurements is used to estimate the system state at one snapshot in time, traditionally by using the weighted least squares method [2]. However, with such an approach, the information contained in the evolution of the system state over consecutive time instants is not taken into account. To this end, [3] and others proposed following the changes of the system by means of quasi-dynamic state estimation. Since this method utilizes forecasting of future values, it is also known as forecasting-aided state estimation (FASE) [4-6]. A typical assumption in this setting is that the system has slowly changing states and that measurement errors can be modeled as Gaussian noise with zero mean and known covariance.

Many state estimation methods developed for the transmission level cannot be implemented at the distribution level without essential changes mainly due to the sparsely

*Correspondence: natallia.makarava@ptb.de

Physikalisch-Technische Bundesanstalt, Abbestr. 2-12, 10587, Berlin, Germany instrumented middle- (MV) and low-voltage (LV) grids $[7,8]$. Consequently, to ensure observability of the system, the so-called pseudo-measurements are required. These pseudo-measurements are usually based on historical data or knowledge about controller set points in the grid and can therefore differ from the real values significantly $[9,10]$. Hence, large uncertainties are associated with their values, which affects the overall state estimation quality. To this end, the so-called nodal load observer (NLO) has been proposed recently [11]. This method is a disturbance observer based on an extended Kalman filter as a dynamic state estimation technique. The NLO aims to correct possibly incorrect pseudo-measurements of bus power and then determines the grid state based on reconstructed and corrected values of nodal power and voltage. That is, the NLO proposes not to use directly the possibly incorrect pseudo-measurements, but rather to make use of all available measurement information. Recent work done on improving pseudo-measurements based on delayed $\mathrm{AMI} / \mathrm{smart}$ meter data can be found in $[12,13]$.

As part of the European research project GridSens ${ }^{1}$, the NLO was investigated regarding its applicability to $\mathrm{LV}$ and $\mathrm{MV}$ grids. Initial investigations using a 
simplified dynamic model and simulated middle-voltage grids showed promising results regarding reconstructed voltage values [14]. However, these studies also concluded that observability and estimation performance could be improved by the implementation of more advanced dynamic models. Thus, we here consider a dynamic model based on autoregressive processes (AR). As for the original simple model, the challenge in its application is the determination of suitable model parameters. Therefore, we consider a recently proposed online estimation method for AR processes based on a recursive maximum likelihood method [15]. We extend the NLO method with an online learning method for the dynamic model parameters, which enables to incorporate the proposed online learning technique to the extended Kalman filter, which results in fast and more reliable estimation results for state estimation in middle- and low-voltage electrical grids. Using a realistic network model and simulated measurement data, we demonstrate the performance of this new method for state estimation in MV and LV electrical grids. The example clearly illustrates that the NLO with adaptive AR model outperforms the NLO estimation with a fixed AR model irrespective of the choice of the initial AR parameter estimates. Hence, the NLO with adaptive AR model is a powerful quasi-dynamic state estimation method for MV and LV electrical grids.

The next two sections are dedicated to the mathematical background of state estimation with some fundamental definitions given in the next section. In Section 3, the AR approach for the NLO is discussed and the online learning technique for its parameters is presented in Sections 3.1 and 3.2. An example of the AR parameter learning integrated within the extended Kalman filter NLO is presented in Section 4. A comparison of the augmented NLO approach to other methods is a topic of future work. For an assessment of the performance of the original NLO, we refer the interested reader to [11] and [14]. For readers interested in an overview of existing power system state estimation methods, we refer to [4].

\section{Background}

A generic system model for quasi-dynamic state estimation is given by

$$
\begin{aligned}
x(k+1) & =F(k) x(k)+g(k)+w(k) \\
z(k) & =h(x(k))+v(k)
\end{aligned}
$$

where $x(k)$ is the state vector, $F(k)$ is the transition matrix, $g(k)$ is the modeling trend behavior, $z(k)$ is the measurement vector, and $h($.$) is the load-flow function. The$ errors $w(k)$ and $v(k)$ are assumed to be independent Gaussian with known covariance matrices $Q(k)$ and $R(k)$, respectively. The extended Kalman filter for this model is then given by [16-18]

$$
\begin{aligned}
\hat{x}(k, k-1)= & F(k) \hat{x}(k-1, k-1)+g(k) \\
P(k, k-1)= & F(k) P(k-1, k-1) F^{T}(k)+ \\
& +Q(k) \\
K(k)= & P(k, k-1) H^{T}(k) \times \\
& \times\left(H(k) P(k, k-1) H^{T}(k)+R(k)\right)^{-1} \\
P(k, k)= & (I-K(k) H(k)) P(k, k-1) \\
\hat{x}(k, k)= & \hat{x}(k, k-1)+ \\
& +K(k)(z(k)-h(\hat{x}(k, k-1)))
\end{aligned}
$$

with Jacobian matrix $H(k)=\left.\frac{\partial h(x(k))}{\partial x(k)}\right|_{x(k)=\hat{x}(k, k-1)}$. Here, Eqs. 3 and 4 denote the Kalman filter prediction step, Eq. 5 the Kalman gain matrix, and Eqs. 6 and 7 the correction step. One advantage of the Kalman filter approach is that estimates of the system states $x(k)$ are obtained together with an estimate of the error covariance $P(k, k)$, which can be interpreted as the uncertainty associated with the system state estimate [19]. The covariance matrices $Q$ and $R$ mainly influence the behavior of the Kalman filter as they model the confidence in the measured values $z(k)$ and the dynamic model values $x(k)$. Here, the entries of the diagonal matrix $Q$ are assumed to be relatively high compared to that of the matrix $R$, which implies that the measurements have a larger impact on the estimation result than the predictions. This also reflects the fact that missing measurement information is replaced by pseudo-measurements.

It is assumed that nodal active and reactive power is either measured or forecasted. Therefore, the vector of bus powers $S(k)$ consists of measured $S^{\text {meas }}(k)$ values and true but unknown $S^{\text {true }}(k)$ values. For a more efficient numerical implementation, this can be expressed as the sum:

$$
S(k)=D_{m} S^{\text {meas }}(k)+D_{n m} S^{\text {true }}(k)
$$

where $D_{n}$ and $D_{n m}$ are possibly non-square matrices with at most one entry equal to 1 in every row, and zeros else. Denoting with $S^{\mathrm{pm}}(k)$ pseudo-measurements at buses without a power measurement, the here considered nodal load observer utilizes then as states $x(k)$ in the dynamic model equation (1) the difference $\Delta S(k)=S^{\text {true }}(k)-$ $S^{\mathrm{pm}}(k)[14]$.

The vector of bus power $S(k)$ is then obtained as a combination of measured bus powers $S^{\mathrm{m}}$ and (improved) pseudo-measurements $S^{\mathrm{pm}}$

$$
S(k)=D_{n m}\left(S^{\mathrm{pm}}(k)+\Delta S(k)\right)+D_{m} S^{\mathrm{m}}(k) .
$$

The matrices $D_{m}$ and $D_{n m}$ contain only zeros and ones and are used to construct the vector of all bus powers by mapping the measurements and pseudo-measurements to the respective bus indices. 
The advantage of this approach is the possible improvement of the pseudo-measurements, whereas the challenge lies in the determination of a suitable dynamic model for the application of the Kalman filter. For the original state-space model in the NLO, the general system 1-2 for quasi-dynamic state estimation is given by [11]:

$$
\begin{aligned}
\Delta S(k+1) & =\gamma \Delta S(k)+w(k) \\
V(k) & =h(\Delta S(k), S(k))+v(k),
\end{aligned}
$$

with $\gamma \in(0,1)$, and the function $h($.$) in the observation$ Eq. 10 is obtained by solving

$$
S=\operatorname{diag}(V) Y V
$$

with respect to $V$, where $V:=(\operatorname{Re}(\bar{V}), \operatorname{Im}(\bar{V}))^{T}$ with $\bar{V}$ denoting a complex nodal voltage and $\operatorname{diag}(V)$ a diagonal matrix with entries containing the nodal voltages. The parameter $\gamma$ has a significant impact on the estimation result as it models how fast systematic errors in the pseudo-measurements are assumed to become solely random noise. For an assessment of the performance of the NLO with this dynamic model, see [11, 14].

State estimation for MV and LV networks requires dealing with three-phase measurements and unbalanced loads. Thus, it is worth noting that the NLO can also be applied for three-phase networks under unbalanced load conditions by replacing Eq. 11 with the corresponding three-phase formulation.

\section{Methods}

In its original definition, the nodal load observer is considered with a simple dynamic model for the deviations of the pseudo-measurements [14]:

$$
\Delta S(k+1)=\gamma \Delta S(k)
$$

with $\gamma \in(0,1)$. However, as already mentioned in [11], more flexible models are required in order to improve the estimation quality of the nodal load observer. Autoregressive moving average models (ARMA) provide a versatile representation of stochastic processes in a sequential way, which is ideal for the use with a Kalman-like filter method. Thus, we here propose their application with the NLO as a means to model the errors in the pseudo-measurements. The benefits of this approach are the possible incorporating of correlation between buses and the improved flexibility for the state estimator.

An $\operatorname{ARMA}(p, q)$ model of order $(p, q)$ consists of two parts, an autoregressive (AR) part of order $p$ and a moving-average (MA) part of order $q$ :

$$
x(k)=\mu+\sum_{i=1}^{p} \varphi_{i} x(k-i)+\sum_{i=1}^{q} \theta_{i} w(k-i)+w(k),
$$

where $w(k)$ is a white noise stochastic process with known variance $E\left[w^{2}(k)\right]=\sigma^{2}(k), \mu$ is the expected value of $x(t)$, and $\varphi_{i}, \theta_{i}$ are the parameters of the AR and MA models, respectively. The original dynamical model Eq. 12 is thus an ARMA $(1,0)$ type model, which can also be written as $\operatorname{AR}(1)$.

Integration of an AR model with the NLO is possible in a straightforward way as part of the state-space model. For instance, considering an $\mathrm{AR}(2)$ model in state space for the disturbance $\Delta S(k)$ and substituting it into the system model Eqs. 9 and 10 with an appropriate replacement, the following system equations are obtained:

$$
\begin{aligned}
\left(\begin{array}{c}
\Delta S(k+1) \\
\Delta S(k)
\end{array}\right) & =\left(\begin{array}{cc}
\varphi_{1} & \varphi_{2} \\
1 & 0
\end{array}\right)\left(\begin{array}{c}
\Delta S(k) \\
\Delta S(k-1)
\end{array}\right)+ \\
& +\left(\begin{array}{c}
\text { const } \\
0
\end{array}\right)+\left(\begin{array}{c}
w(k) \\
0
\end{array}\right) \\
V(k) & =h(\Delta S(k), S(k))+v(k) .
\end{aligned}
$$

It is well known that for linear state-space models, the Kalman filter produces optimal estimates for AR-like state evolution models [20]. In principle, the extended Kalman filter can be applied in the same way for non-linear measurements with AR-like state evolutions. However, care has to be taken regarding the convergence of the resulting Kalman filter estimation, e.g., by means of carefully choosing suitable AR coefficients. That is, for certain sets of AR coefficients, the state estimation result can diverge indefinitely. In practice, this may require a repeated application of the extended Kalman filter with different choices for the AR coefficients. However, only significant deviations from an optimal choice of coefficient could be determined in such a way as the true value remains unknown. This would make the practical application of the NLO with AR dynamic model almost impossible. Therefore, we here propose the incorporation of recently developed online learning algorithms for AR processes with the extended Kalman filter of the NLO.

\subsection{Online parameter learning}

Online or recursive estimation methods for parametric stochastic processes appeared in the middle of the 20th century to replace the so-called offline methods in which first all data is collected and then the model parameters are estimated. Offline estimation approaches are less efficient due to expensive computation time costs, power, and memory. This is why algorithms which allow estimating the model parameters when new data is available during the operation are applied in different areas of engineering (see for example [21, 22]). These methods are typically called online.

An investigation of various recursive estimation methods available from the literature showed the recursive maximum likelihood (RML) method originally presented by [23] to be most suitable for an application with the NLO. Several modifications of this method can be found 
in the literature $[15,24]$. Our analysis is based on the algorithms introduced in [15], where also a proof of convergence is given.

Consider real-valued observations $\left\{x_{t} ; t=1, \cdots, N\right\}$ of an $\operatorname{ARMA}(p, q)$ process as defined in Eq. 13 and let $\beta=\left(\varphi_{1}, \cdots, \varphi_{p}, \theta_{1}, \cdots, \theta_{q}\right)^{T}$ be the vector of sought coefficients with the corresponding estimate at time $t$ being: $\beta_{t}=\left(\hat{\varphi}_{1, t}, \cdots, \hat{\varphi}_{p, t}, \hat{\theta}_{1, t}, \cdots, \hat{\theta}_{q, t}\right)^{T}$. For a given $\beta_{t}$, the forecast $\hat{x}_{t}\left(\beta_{t}\right)$ can be computed from the ARMA model definition by using the residuals $\varepsilon_{t-1}=x_{t-1}-\hat{x}_{t-1}\left(\beta_{t-1}\right)$ as the driving noise $w_{t}$.

Let denote the opposite of the derivative of the residuals $\varepsilon_{t}\left(\beta_{t}\right)$ with respect to $\beta_{t}$ by the vector $\psi_{t}\left(\beta_{t}\right)=$ $-\left[\frac{\partial \varepsilon_{t}\left(\beta_{t}\right)}{\partial \beta_{t} T}\right]^{T}$. The elements of this vector can be calculated analytically from the definition of $\varepsilon$ and $\beta_{t}$ [15].

Adopting the algorithms from [15], we derive the algorithm for the estimation of $\beta$ as follows:

1. With $\phi_{t}^{T}=\left(x_{t}, \cdots, x_{t-p+1},-w_{t}, \cdots, w_{t-q+1}\right)$ update the gradient by

$$
\psi_{t}=\sum_{k=1}^{q} \hat{\theta}_{k, t-1} \psi_{t-k}+\phi_{t-1} ;
$$

2. Calculate the forecasting error as

$$
\varepsilon_{t}=x_{t}-\beta_{t-1}^{T} \phi_{t-1}
$$

3. Update the estimate of $\beta_{t}$ using the quasi-Newton step

$$
\beta_{t}=\beta_{t-1}+\gamma_{t} \hat{\sigma}_{t}^{-2} I \psi_{t} \varepsilon_{t},
$$

with $I$ denoting the identity matrix.

4. Update the estimate of the ARMA noise process variance $\sigma^{2}$ by

$$
\hat{\sigma}_{t+1}^{2}=\hat{\sigma}_{t}^{2}+\gamma_{t}\left(\varepsilon_{t}^{2}-\hat{\sigma}_{t}^{2}\right) .
$$

Figure 1 illustrates the application of the above estimation algorithm for an $\mathrm{AR}(2)$ process with true parameters being $\varphi_{1}=1.15, \varphi_{2}=-0.15$, and $\sigma^{2}=0.01$. For this result, we used the following initial values: $\hat{\varphi}_{1,0}=$ $1.5, \hat{\varphi}_{2,0}=-0.5$, and $\hat{\sigma}_{0}^{2}=10$. We generated 10,000 realizations of length $t=100$ and computed estimates of $\varphi$ with $\gamma_{t}=\frac{1}{t}$ for each realization. The obtained results clearly illustrate the convergence of the method to the true value, which empirically confirms the proof of convergence from [15].

\subsection{NLO with online learning technique}

In this section, we present the final algorithm, which is obtained by combining the basic procedure of the extended Kalman filter, the nodal load observer for power distribution grids, and the considered online learning technique for AR process parameters.
Initial values $\hat{x}(0), P(0), \hat{V}(0), \beta(0)$, and $\sigma(0)$ are chosen, and the matrix $C_{m}$ set up with one entry equal to 1 in every row such that $V_{m}=C_{m} V$, with $V$ as the vector of all nodal voltages in rectangular coordinates and $V_{m}$ as the corresponding measured values. The NLO algorithm [14] with adaptive dynamic model [15] can then be written as follows.

The Kalman prediction step is given by

$$
\begin{aligned}
\hat{x}(k, k-1) & =\left(\begin{array}{cc}
\varphi_{1} & \varphi_{2} \\
1 & 0
\end{array}\right)\left(\begin{array}{l}
\hat{x}(k-1) \\
\hat{x}(k-2)
\end{array}\right) \\
P(k, k-1) & =\left(\begin{array}{cc}
\varphi_{1} & \varphi_{2} \\
1 & 0
\end{array}\right) P(k-1)\left(\begin{array}{ll}
\varphi_{1} & 1 \\
\varphi_{2} & 0
\end{array}\right)+ \\
& +Q(k, \sigma) .
\end{aligned}
$$

With initial values $\eta(0)=\hat{x}(k, k-1), v(0)=\hat{V}(k-1)$, and $j=0$ for the measurement update iteration, an estimate of nodal voltages is then obtained from the power flow (PF) calculation:

$v(j+1)=\operatorname{PF}\left(D_{m} S_{m}(k)+D_{n m}\left(S^{\mathrm{pm}}(k)+\eta(j)\right), V(k), v(j)\right)$.

With $H(k, j)$

$$
H(k, j)=C_{m}\left(\left.\frac{\partial}{\partial \nu} \operatorname{diag}(v) Y v\right|_{v=v(j+1)}\right)^{-1} D_{n m}
$$

the Kalman gain is calculated as

$$
\begin{aligned}
K(k, j)= & P(k, k-1) H^{T}(k, j) \times \\
& \times\left(H(k, j) P(k, k-1) H^{T}(k, j)+R(k)\right)^{-1},
\end{aligned}
$$

which yields the updated estimate of the errors in the pseudo-measurements as

$$
\begin{aligned}
\hat{x}(k)= & \hat{x}(k, k-1)+K(k, j) \times \\
& \times\left[V_{m}(k)-C_{m} v(j+1)-H(k, j)(\hat{x}(k, k-1)-\eta(j))\right],
\end{aligned}
$$

with updated covariance matrix given by

$$
P(k)=(I-K(k, j-1) H(k, j-1)) P(k, k-1) .
$$

The updated estimates of bus power and nodal voltage are then calculated as

$$
\begin{aligned}
\hat{S}(k) & =D_{m} S_{m}(k)+D_{n m}\left(\hat{x}(k)+S^{\mathrm{pm}}(k)\right) \\
\hat{V}(k) & =v(k) .
\end{aligned}
$$

Utilizing the updated state estimates, the AR model parameters are then updated as follows:

$$
\begin{aligned}
\psi(k) & =(\hat{x}(k-1), \hat{x}(k-2))^{T} \\
\varepsilon(k) & =\hat{x}(k)-\hat{\beta}(k-1)^{T}[\hat{x}(k-1), \hat{x}(k-2)]^{T}, \\
\hat{\beta}(k) & =\hat{\beta}(k-1)+\gamma(k) \hat{\sigma}^{-2}(k) I_{2} \psi(k) \varepsilon(k), \\
\hat{\sigma}^{2}(k+1) & =\hat{\sigma}^{2}(k)+\gamma(k)\left(\varepsilon^{2}(k)-\hat{\sigma}^{2}(k)\right),
\end{aligned}
$$

with damping sequence $\gamma(k)=\frac{1}{k}$.

The selection of initial values for the online learning of AR parameters is an important aspect in the estimation algorithm. For example, the initial value for the noise 

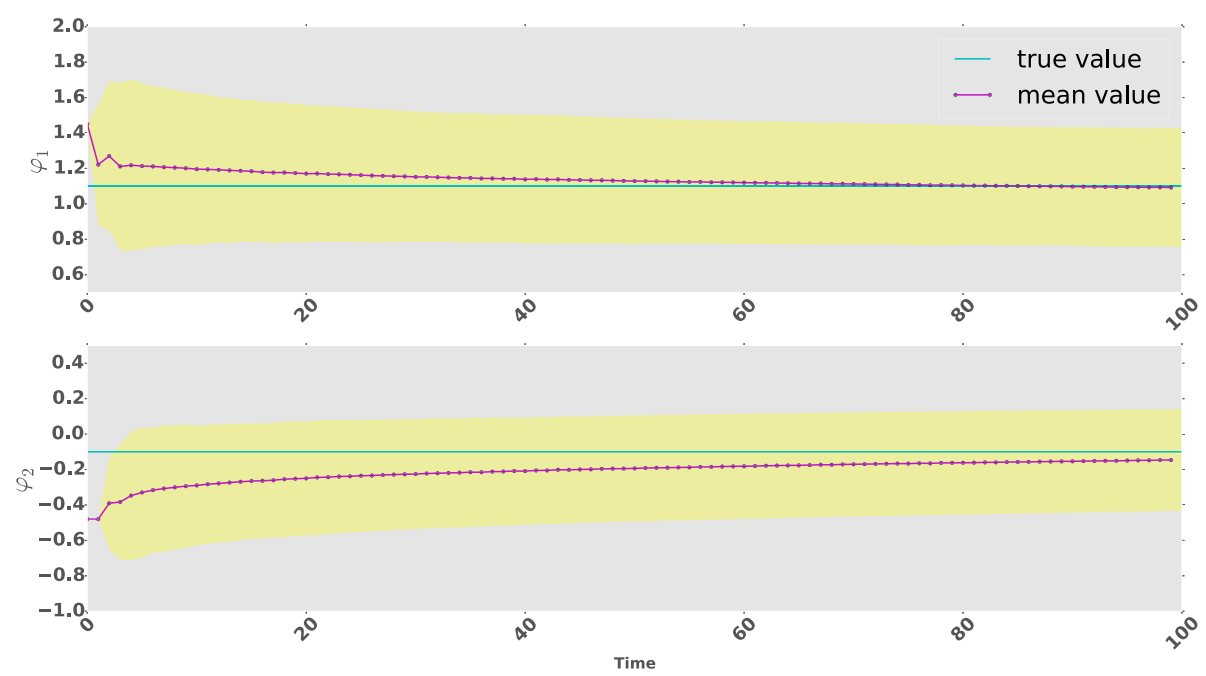

Fig. 1 Estimation results of ARMA $(2,0)$ process with parameters $\varphi=[1.15,-0.15]$ and $\sigma^{2}=0.01$ for initial value parameters $\varphi=[1.5,-0.5]$ and $\sigma^{2}=10$ with 10,000 realizations and length of the data $t=100$. With yellow color, the area of standard deviation is shown

variance $\sigma_{t}^{2}$ is preferable to be taken bigger than needed with expectation to convert to the innovation variance $\frac{1}{t} \sum_{k=1}^{t} \varepsilon_{t}^{2}(\beta)$. The second significant issue is the choice of $\gamma$. Theoretically, it is recommended to choose $\gamma_{t}=\frac{1}{t}$, although in practice, it should be selected such in a way to improve the convergence. The authors in [15] proposed to define $\gamma_{t}$ through the "forgetting" factor $\lambda_{t}$ as follows:

$$
\gamma_{t}=\frac{\gamma_{t-1}}{\lambda_{t}+\gamma_{t-1}}
$$

with

$$
\lambda_{t}=\lambda^{0} \lambda_{t-1}+\left(1-\lambda^{0}\right),
$$

while different values of $\lambda^{0}$ can be chosen. In the example shown in Section 4, $\gamma_{0}=1.0$ and $\lambda_{0}=1.0$ with $\lambda^{0}=0.99$ were identified as good choice based on validation tests made for different $\varphi$ values.

In order to obtain initial estimates of suitable AR parameters, we fitted an $\mathrm{AR}(2)$ process to the difference $\Delta S(k)=$ $S^{\text {true }}(k)-S^{\mathrm{pm}}(k)$ at a bus with measured bus power available.

\section{Results and discussion}

To illustrate the proposed adaptive dynamic state estimation method, we consider a grid model developed by a consortium of UK universities specifically for testing purposes (UKGDS). The grid presented in Fig. 2 is a $11-\mathrm{kV}$ urban network fed from a 33-kV supply point and has 12 buses, 11 branches with a generator at bus number 5 , and the slack bus has number 0 . This grid is a part of the original 77 buses grid, which due to the radial topology can be examined independently [14]. This grid was chosen, because it has been studied for the original NLO before to demonstrate its performance [14].
Measurements in this network have been simulated based on realistic load profiles. Therefore, a timedependent generator output was assumed, and voltages calculated at every time step with the open-source software PyPower for optimal power flow estimation. The

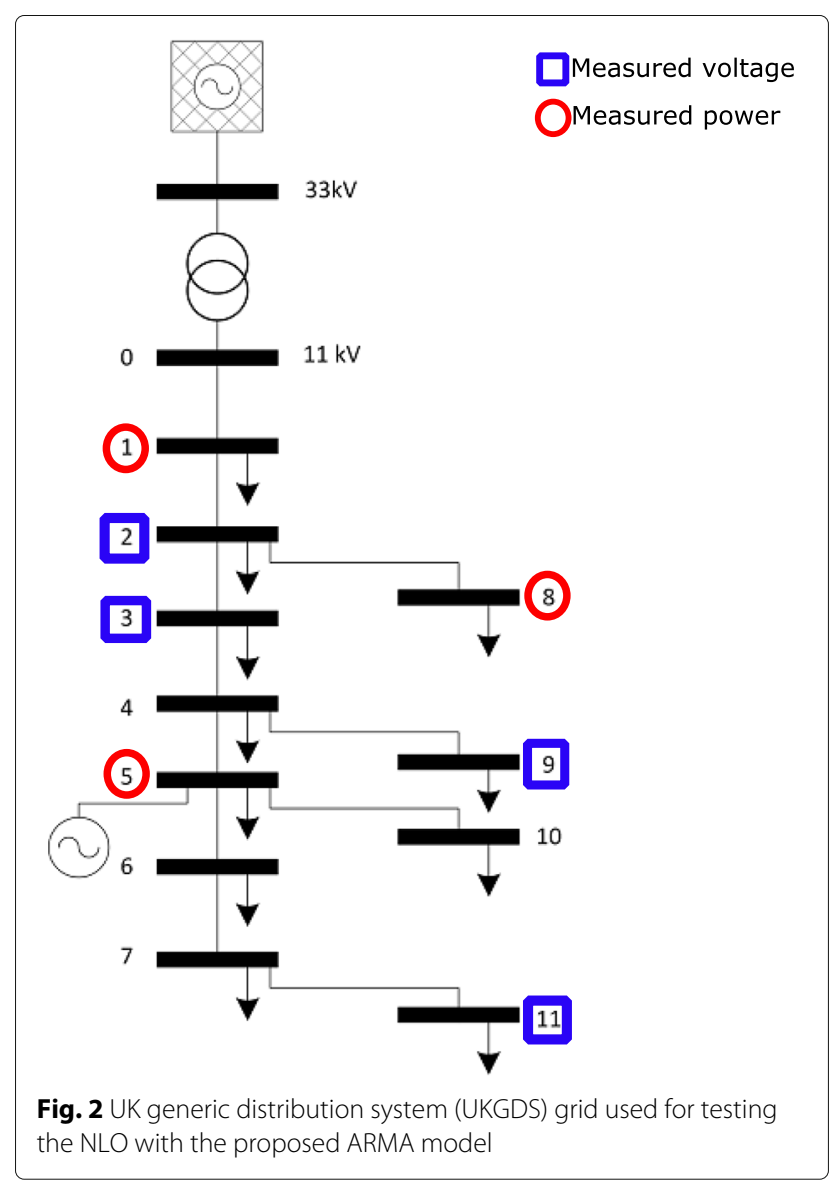


pseudo-measurements were then constructed by combining simulated data and load data.

In the scenario studied here, measurement of active and reactive power is simulated for buses 1,5 , and 8; measured voltage is simulated at buses $2,3,9$, and 11 , shown in Fig. 2 with blue squares and red circles, respectively. The data set represents the course of one day with measurements taken every $15 \mathrm{~min}$, resulting in 96 data points. This small number of consecutive measurements is typical for such networks, but problematic for many online learning techniques due to the required speed of convergence.

The results of the application of the proposed extended Kalman filter with online learning for the estimation of the parameters of an AR(2) dynamic model are shown in Fig. 3 for bus 6. As initial parameters for the AR(2) dynamic model, we chose $\varphi=[1.45,-0.5]$ and $\sigma=$ 0.001 . Compared to the pseudo-measurements, the estimated power obtained by the NLO with online learning clearly performs better than the original NLO with fixed parameters. Moreover, as shown in Fig. 3, the estimation results improve over time as the online learning technique improves the estimate of the $\mathrm{AR}(2)$ process parameters. On the right-hand side of Fig. 3, the estimation error for all three estimation approaches is presented. This shows that the online learning technique needs some time to train the model parameters, but then outperforms the other two approaches. The original NLO estimates the true value of power very accurately at the beginning of the time series, but shows less efficiency for the main part. In contrast, the NLO with AR model with fixed parameters is almost identical to the pseudo-measurements.

The results demonstrate two findings: (i) A simple extension of the NLO dynamic model to an AR model is not sufficient, but requires a sensitive choice of model parameters. (ii) The online learning method for the AR model parameters offers a very efficient solution to this task and clearly outperforms the original NLO with its simple dynamic model. In Fig. 4, the change of the model parameters in the application of the adaptive NLO method is shown. The change from the original values is $\max _{t} \mid \varphi(t)-$ $\hat{\varphi}(t) \mid=(0.00142,0.00138)$ and thus fairly small. Nevertheless, these changes play a significant role in the improvement of the estimation results. Hence, a manual selection of suitable parameters is hardly possible. The situation is similar at the other buses in the network and for the estimation of reactive power. This is presented on Fig. 5 for the node 4 . With regard to the computational costs, for both approaches, the approximate time of estimation of 12 buses electrical grids was less than $5 \mathrm{~s}$ on a standard desktop PC and thus sufficient for an application in practice.

In order to emphasize the advantage of the online learning technique over the utilization of fixed parameters, we carried out a simulation study for a wide range of AR coefficients $\beta=\left(\varphi_{1}, \varphi_{2}\right)$. We considered $\varphi_{1} \in[1.0,1.9]$ and $\varphi_{2} \in[-0.9,-0.1]$ with steps equal to 0.1 and compared the root-mean-square (RMS) deviation between estimated and true values of active power for the aforementioned bus number 6 along the time. The results are shown in Fig. 6. On the left-hand side of Fig. 6 are shown the logarithmic RMS deviations for the online learning technique, whereas in the right-hand side figure, the results for the fixed parameters model are shown. It is clearly visible that there are two distinct regions. In the upper left area, the results are very similar for both approaches which can be explained by the fact that for all $\varphi_{1}$ and $\varphi_{2}$ from this range, the results of the online learning technique stayed almost unchanged over time. The opposite picture is met in the bottom right area. There, it is clearly visible that the RMS errors for the
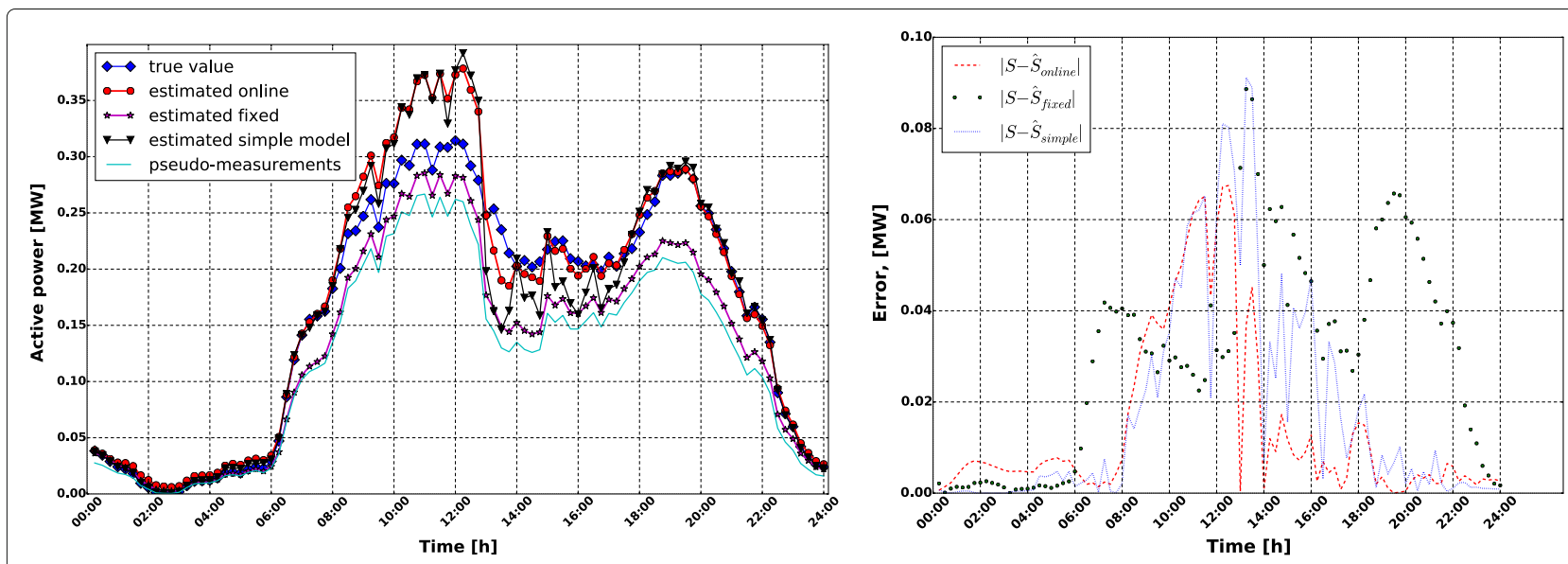

Fig. 3 Left: power estimation using the NLO with the proposed online learning technique, using the NLO with fixed ARMA(2,0) parameters and the (simulated) true values and pseudo-measurements considered. Right: error between true values of power and estimated with three models: simple model, $\operatorname{ARMA}(2,0)$ model with fixed parameters, and $\operatorname{ARMA}(2,0)$ with online learning technique 


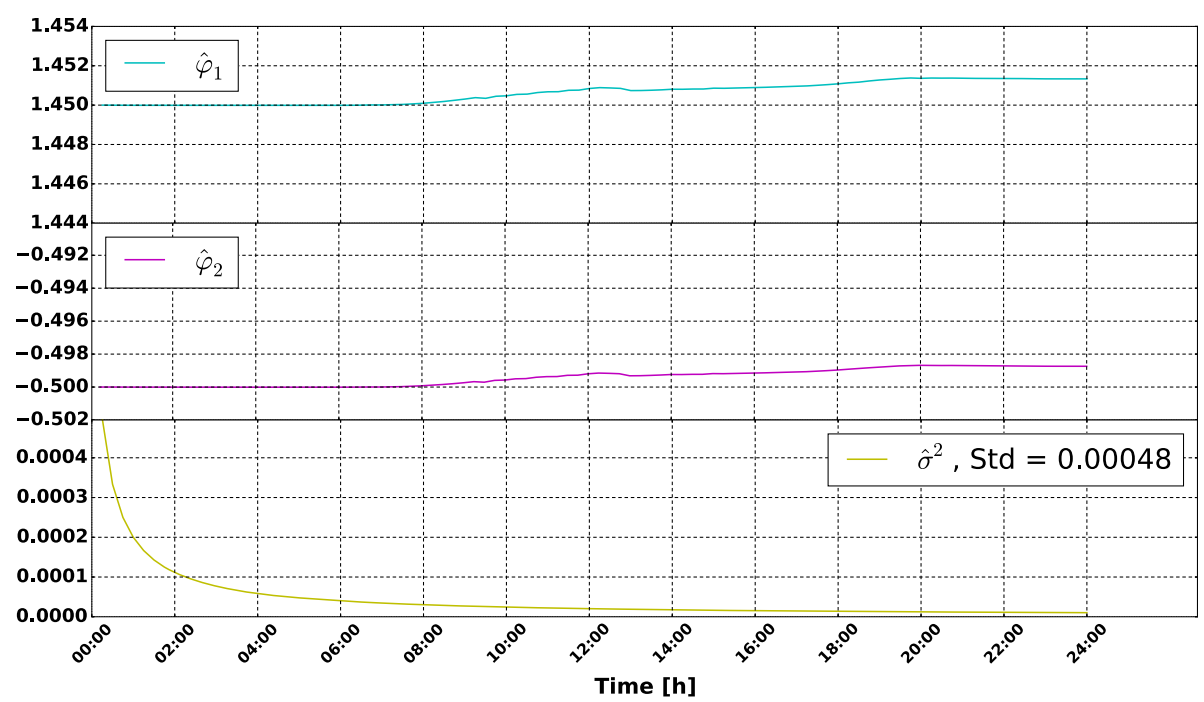

Fig. 4 Evolution of the online estimation of the ARMA coefficients $\varphi$ and $\sigma$. Standard deviation for $\sigma=0.00048$

fixed parameters approach are significantly larger than those obtained by the online learning technique, indicating convergence of the estimated AR parameters. Again, a similar situation holds for the other buses where only pseudo-measurements of bus power where available for the estimation. The measurement uncertainties concerning the Kalman filter for state estimation could be found in [19].

For a particular choice of initial AR parameter estimates, Fig. 7 presents the time evolution of the estimation errors for active power at bus 6 with $\varphi=(1.7,-0.3)$. Here, the left-hand side figure shows the estimation error over time for the NLO with fixed AR parameters. The red dashed line in right-hand side figure shows the results for the NLO with the online learning technique with yellow dots showing the change of $\varphi$. It is clearly visible in Fig. 7 when the parameters $\varphi$ start varying and how this interacts with the estimation. That is, the NLO with online learning shows an adaption to the change in the estimation quality, whereas the error for the NLO with fixed parameters increases exponentially. This corresponds to the situation shown in Fig. 3, where the estimation quality for certain choices of the AR parameters results in a almost arbitrarily large RMS estimation errors for the NLO with fixed AR parameters whereas the adaptive NLO shows a much better performance in the same situation.

\section{Conclusions and outlook}

It has been shown previously that the original nodal load observer (NLO) approach to state estimation in LV and MV grids has the principle advantage of improving over the pseudo-measurements by estimating the remaining

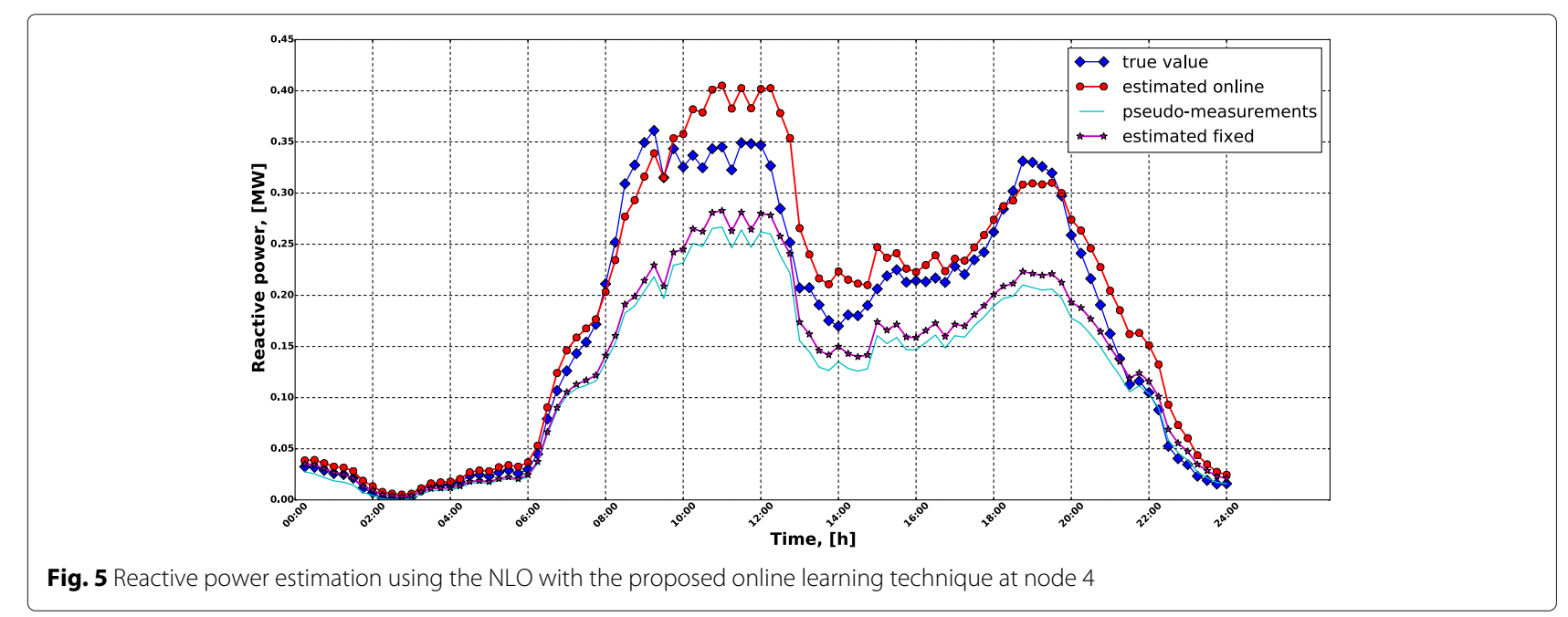



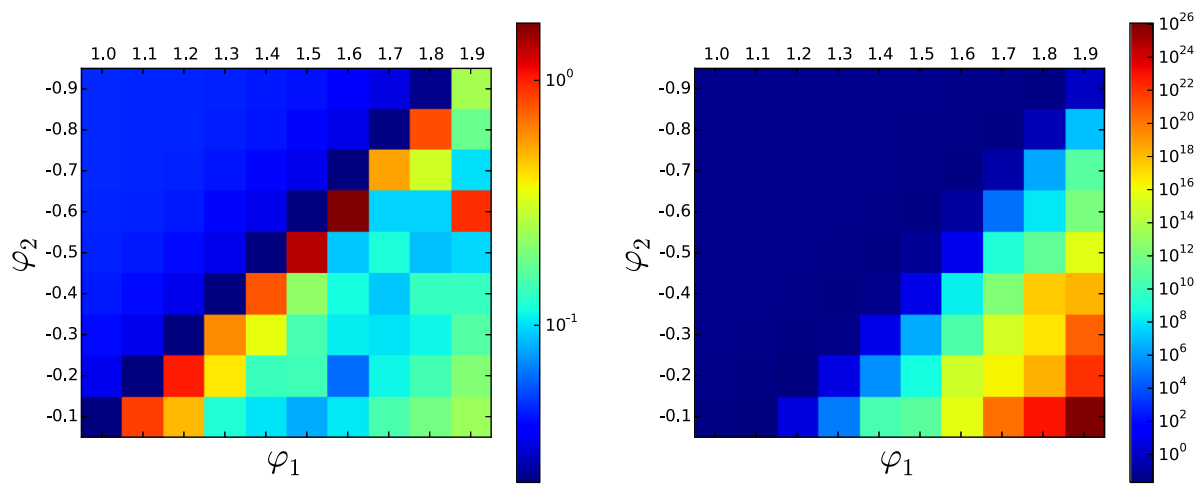

Fig. 6 Root-mean-square deviation between estimated and true values of active power at bus 6 for various initial ARMA(2,0) parameters $\left(\varphi_{1}, \varphi_{2}\right)$ Left: Results for the NLO with online learning technique. Right: Results for the NLO with fixed ARMA $(2,0)$ parameters

error using a Kalman filter. However, it has also been discussed in previous publications that there are a number of issues which have to be overcome in order to yield reliable state estimates for practical networks. The main drawback of the original NLO is the simple dynamic model. To this end, we here proposed a versatile dynamic model for an improved estimation of the pseudo-measurements errors based on an AR process model. The challenge in the application of AR processes with non-linear state estimation is in the choice of AR model parameters. Therefore, we considered a recently proposed online learning technique for the estimation of AR parameters and integrated this into the NLO. The online learning method calculates an update to the AR model parameters in each time step using, and can thus be applied in parallel to the Kalman filter. Estimation of AR parameters were received running over 96 values for all 11 buses (except the slack bus). That is, the method recalculates the AR model parameters online in each time step instead of for the whole time series in a batch approach.
With this approach, the NLO for the first time is able to produce accurate and fast results for state estimation of electrical power grids. The advantage of using a recursive technique for model parameter estimation was demonstrated by a comparison with the NLO with fixed AR parameters for a realistic MV distribution network. We demonstrated the adaptive nature of the online learning technique and its performance compared to using a fixed AR model. The results clearly showed that already small changes to the model parameters can have a significant impact and that the online learning technique is able to adjust the AR model parameters with very good rate of convergence.

The proposed extension of the NLO results in a robust, reliable state estimator for MV and LV electrical grids. Our initial test shown here demonstrated the potential of this novel approach. It can be expected that this also holds for AR models of higher dimension and larger networks. This will be a topic of future research. Initial tests with actual measurement data of electrical MV networks
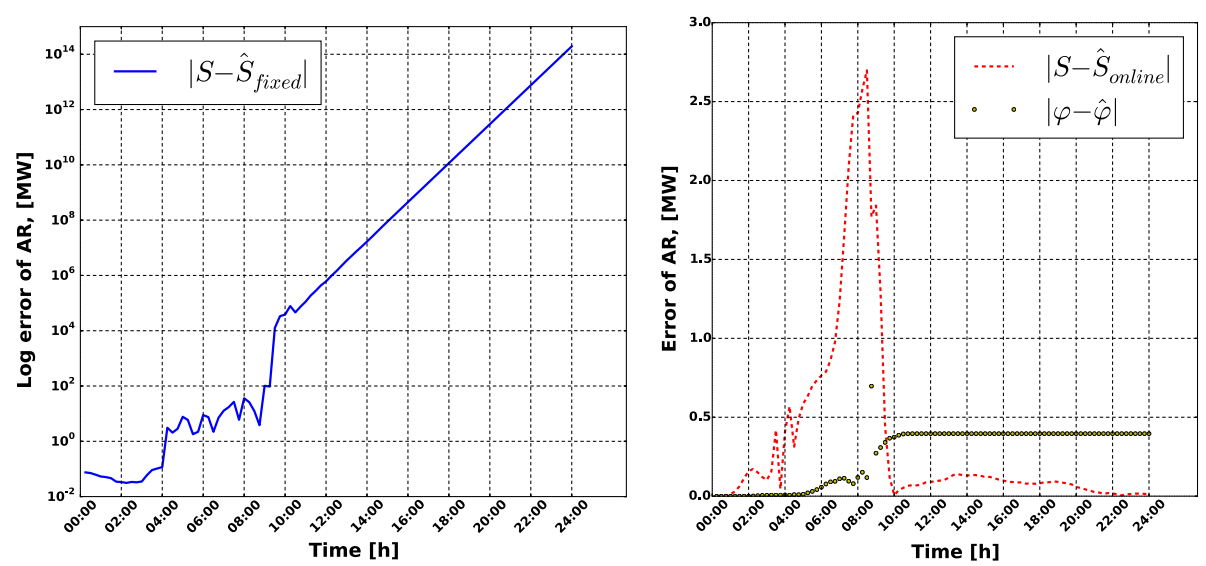

Fig. 7 Error between estimated and true values of active power at bus 6 for $\varphi=[1.7,-0.3]$. Left figure shows the error for the fixed parameters model, whereas in the right one the red dashed line corresponds to the results for online learning technique and yellow dots show the error of $\varphi$ 
furthermore showed promising results and will be a topic of a future work. Moreover, the incorporation of weather forecasts for improved pseudo-measurements, uncertainty of line impedances, and other topology parameters as well as other kinds of measurement data with the NLO will be addressed in future research.

\section{Endnote}

${ }^{1}$ www.gridsens.eu

\section{Acknowledgment}

We would like to thank Eindhoven University of Technology for providing the photovoltaic data.

\section{Abbreviations}

AR: Autoregressive processes; ARMA: Autoregressive moving average models; EMRP: European Metrology Research Program; FASE: Forecasting-aided state estimation; LV: Low-voltage; MV: Middle-voltage; NLO: Nodal load observer; RML: Recursive maximum likelihood; RMS: Root-mean-square; UKGDS: UK generic distribution system

\section{Authors' contributions}

All authors contributed equally to this work. All authors read and approved the final manuscript.

\section{Funding}

This work was financially supported by the European Metrology Research Program (EMRP) Joint Research Project ENG63. The EMRP is jointly funded by the EMRP participating countries within EURAMET and the European Union.

\section{Availability of data and materials}

Please contact author for data requests.

\section{Ethics approval and consent to participate}

Not applicable.

\section{Consent for publication}

Not applicable.

\section{Competing interests}

The authors declare that they have no competing interests.

Received: 11 February 2019 Accepted: 28 June 2019

Published online: 28 August 2019

\section{References}

1. A. Abur, A. Gómez-Expósito, Power system state estimation: theory and implementation. (CRC Press, Parent Company Taylor \& Francis, United States, 2004)

2. J. Wan, K. N. Miu, Weighted least squares methods for load estimation in distribution networks. IEEE Trans. Power Syst. 18(4), 1338-1345 (2003)

3. A. S. Debs, R. E. Larson, A dynamic estimator for tracking the state of a power system. IEEE Trans. Power Appar. Syst. PAS-89(7), 1670-1678 (1970)

4. M. B. Do Coutto Filho, J. C. Stacchini de Souza, Forecasting-aided state estimation — part i: panorama. IEEE Trans. Power Syst. 24(4), 1667-1677 (2009)

5. M. B. Do Coutto Filho, J. D. Glover, A. M. Leite da Silva. State Estimators with Forecasting Capability. 11th Power systems computation, Avignon, France. vol. 2. (Power Systems Computation Conference, Avignon, 1993), pp. 689-695

6. A. M. Leite da Silva, M. B. Do Coutto Filho, J. F. de Queiroz, State forecasting in electric power systems. IEE Proc. C - Gener. Transm. Distrib. 130(5), 237-244 (1983)

7. J. Huang, V. Gupta, Y.-F. Huang, Electric State Estimators for Distribution Systems with Microgrids, (2012)

8. R. Singh, B. C. Pal, R. A. Jabr, Choice of estimator for distribution system state estimation. IET Gener. Transm. Distrib. 3(7), 666-678 (2009)
9. J. Wu, Y. He, N. Jenkins, A robust state estimator for medium voltage distribution networks. IEEE Trans. Power Syst. 28(2), 1008-1016 (2013)

10. B. P. Hayes, J. K. Gruber, M. Prodanovic, A closed-loop state estimation tool for mv network monitoring and operation. IEEE Trans. Smart Grid. 6 , 2116-2125 (2015)

11. W. Heins, G. Gewiss, C. Bohn, H.P. Beck. in Proceedings of the XXIMEKO World Congress, State-space Formulation for the Nodal Load Observer for Smart Electrical Grids with Imperfect Measurement Infrastructure. (XX IMEKO World Congress, 2012), pp. 1-6

12. B. P. Hayes, M. Prodanovic, State forecasting and operational planning for distribution network energy management systems. IEEE Trans. Smart Grid. 7(2), 1002-1011 (2016)

13. U. Singh, V. Zamani, M. Baran, in 2016 IEEE Power and Energy Society General Meeting (PESGM). On-line load estimation for distribution automation using ami data (IEEE, Piscataway, 2016), pp. 1-5

14. W. Heins, N. Ell, H.P. Beck, C. Bohn. in Proceedings of the 13th European Control Conference, State Observation in Medium-voltage Grids with Incomplete Measurement Infrastructure Through Online Correction of Power Forecasts. (IEEE, 2014), pp. 1306-1312

15. A. Ouakasse, G. Mélard, On-line estimation of arma models using fisher-scoring. Syst. Sci. Control Eng. 2(1), 406-432 (2014)

16. M. Boutayeb, H. Rafaralahy, M. Daraouach, Convergence analysis of the extended kalman filter used as an observer for nonlinear deterministic discrete-time series. IEEE Trans. Autom. control. 42(4), 581-586 (1997)

17. S. J. Julier, J. K. UhImann, in Proceedings of the IEEE. Unscented filtering and nonlinear estimation (IEEE, Piscataway, 2004), pp. 401-422

18. G. Valverde, V. Terzija, Unscented kalman filter for power system dynamic state estimation. Gener. Transm. Distrib. IET. 5, 29-37 (2011)

19. S. Eichstädt, N. Makarava, C. Elster, On the evaluation of uncertainties for state estimation with the Kalman filter. Meas. Sci. Technol. 27(12) (2016)

20. J. Hamilton, in Handbook of Econometrics, 1st edn, ed. by R. F. Engle, D. McFadden. State-space models, (chapter 50), vol 4 (Elsevier, 1986), pp. 3039-3080

21. S. Bhatnagar, H. L. Prasad, L. A. Prashanth, Stochastic Recursive Algorithms for Optimization. (Springer, London, 2013)

22. V. Popovskij, A. Barkalov, L. Titarenko, Control and Adaptation in Telecommunication Systems. (Springer, Berlin Heidelberg, 2011)

23. L. Ljung, Analysis of recursive stochastic algorithms. IEEE Trans. Autom. control. 22(4), 551-575 (1977)

24. O. Anava, E. Hazan, S. Mannor, O. Shamir, in COLT 2013 - The 26th Annual Conference on Learning Theory, June 12-14, 2013, Princeton University, NJ, USA. Online learning for time series prediction, (2013), pp. 172-184

\section{Publisher's Note}

Springer Nature remains neutral with regard to jurisdictional claims in published maps and institutional affiliations.

\section{Submit your manuscript to a SpringerOpen ${ }^{\circ}$ journal and benefit from:}

- Convenient online submission

- Rigorous peer review

- Open access: articles freely available online

- High visibility within the field

- Retaining the copyright to your article

Submit your next manuscript at $\boldsymbol{\nabla}$ springeropen.com 